\title{
Resolving the Controversial Issues and Optimal New Protocols for Accelerated Corneal Cross-Linking (CXL)
}

\author{
Jui Teng Lin* \\ Chairman \& CEO of New Vision Inc Taipei, Taiwan \\ *Corresponding author: Jui Teng Lin, Chairman \& CEO of New Vision Inc Taipei, Taiwan.
}

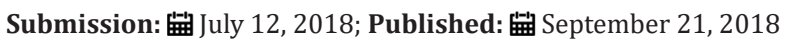

\begin{abstract}
The debating issues of corneal collagen cross-linking (CXL) will be resolved/analyzed mathematically including: the validation of Bunsen Roscoe law (BRL), the intensity dynamics and its cutoff maximum, the safety criteria, the demarcation depth, and the role of oxygen. In addition to a review, the new protocol using concentration-controlled method will be updated. A new CXL efficacy defined by the product of [strength] (or the maximal value of S) and the [depth] (or $z^{*}$ ), i.e., the volume of stroma being cross-linked is introduced. CXL efficacy is shown proportionally related to the demarcation line depth, and both have a cutoff maximum intensity.
\end{abstract}

Keywords: Corneal crosslinking; Corneal keratoconus; Efficacy; Kinetic modeling; Oxygen; Riboflavin; Ultraviolet light; Photodynamic therapy

Abbreviations: CXL: Corneal Collages Cross-linking; CCM: Controlled-Concentration-Method; RF: Riboflavin; BRL: Bunsen Roscoe Law; AXL: Accelerated CXL; OM: Oxygen-Mediated; NOM: Non-Oxygen-Mediated; PS: Photo Sensitizers; DXL: Dresden Protocol

\section{Introduction}

Corneal collages cross-linking (CXL) is a clinical procedure using UVA light (at $365 \mathrm{~nm}$ ) to activate riboflavin solution causing biomechanical properties change of the corneal stroma for the treatment of keratoconus, or producing free oxygen radicals to treat corneal ulcers. Other potential applications include the reduction of postoperative regression in vision correction and scleral treatment in malignant myopia, scleromalacia and low tension glaucoma. The first animal data was reported by Wollensak [1] in 2003 for the treatment of keratoconus. Extensive clinical aspects of CXL have been covered in a recent book edited by Hafezi and Randleman [2]. The theoretical aspects of CXL analyzing the kinetics and fundament formulas have been developed by Lin [3-13]. This Editorial Review will address the current controversial issues in CXL with possible theoretical resolutions. It will also update (with greater details) the new protocol called controlled-concentration-method (CCM) proposed by Lin [11].

The controversial issues to be discussed include:
A. Safety criteria (and the minimum corneal thickness
B. Dynamic profiles and depletion of riboflavin
C. Validation of Bunsen Roscoe law (BRL)
D. UV light Intensity cutoff maximum and minimum exposure time
E. The role of oxygen and pulsed mode
F. CXL efficacy (type-I and type-II)
G. Dresden vs. Modern protocols
H. Demarcation line depth vs. CXL efficacy

\section{Controversial Issues}

\section{New safety criteria $[3,7]$}

The conventional accepted safety criteria for minimum corneal thickness $\mathrm{z}^{*}=400 \mathrm{um}$, for a dose of $5.4 \mathrm{~J} / \mathrm{cm}^{2}$, proposed by Wollensak et al. [1] was meaningless without specifying the riboflavin (RF) concentration, $\mathrm{C} 0$, and its profile, besides its underestimated the endothelium damaged threshold (Ed) at least a factor of 8 according to the clinical data of Mooren et al. [14]. The minimum corneal thickness formula is given by [7] $\mathrm{z}=(1 / \mathrm{A})\left[2.2+\ln \left(\mathrm{E} / \mathrm{E}^{\prime}\right)\right]$, where $\mathrm{A}$ is an effective absorption given by $A=290 \mathrm{~m}\left(1-0.25 \mathrm{z} / \mathrm{D}\right.$ ) $\mathrm{C}_{0}+32$ (with fit parameter $\mathrm{m}$ ). We note that $\mathrm{z}$ is a decreasing function of $\mathrm{C}_{0}$ and $\mathrm{D}$ and increasing function of the UV dose ratio E/Ed, At the referenced point, $E=E^{\prime}, z^{*}=400 u m$, as expected. The conventional safety criteria [1], $E=5.4 \mathrm{~J} / \mathrm{cm}^{2}$ and $\mathrm{z}=400 \mathrm{um}$, is just one of the special case, for $\mathrm{Ed}=0.35 \mathrm{~mW} / \mathrm{cm}^{2}$ (under the Dresden protocol) and cannot be the safety standard. 


\section{Dynamic profiles}

Conventional modeling [15-18] assumed a constant RF concentration during the crosslink, which is true only under the so-called Dresden protocol $[1,2]$, in which the RF is constantly resupplied to compensate its depletion. However, it also reduced the available effective dose to approximately about $70 \%$ to $80 \%$ of the applied dose $5.4 \mathrm{~J} / \mathrm{cm}^{2}$. The constant-RF also underestimated the UV light intensity, which in general, is an increasing function of time (when RF depletion is included), given by $[5,6] \mathrm{I}(\mathrm{z}, \mathrm{t})=\mathrm{I}_{0} \exp [-\mathrm{A}(\mathrm{z}, \mathrm{t})]$ with $A(z, t)$ is a decreasing function of time when $C(z, t)$ is depleted, given by $A(z, t)=2.3\left[(a-b) C(z, t) G(z)+b C_{0}\right]+Q$, where $a, b$ and $Q$ are, respectively, the absorption constant of $\mathrm{RF}$, photolysis product and stroma (without RF); $G(z)=1-0.25 z / D$; and $F(z)=1-0.5 z / D$ is the initial RF distribution defined by a diffusion constant (D) [6].

The dynamic profiles of $\mathrm{RF}$ and concentration and UV light intensity are shown, where $\mathrm{C}(\mathrm{z}, \mathrm{t})$ is a decreasing function (depletion) of time, whereas $\mathrm{I}(\mathrm{z}, \mathrm{t})$ is an increasing function of time due to reduced absorption, $\mathrm{A}(\mathrm{z}, \mathrm{t})$. Crosslink time, $\mathrm{T}^{*}(\mathrm{z}, \mathrm{t})$, defined by the when $\mathrm{C}(\mathrm{z}, \mathrm{t})$ is depleted to 0.13 , and is given by $\mathrm{T}^{*}=\mathrm{T}_{0} \exp (\mathrm{Az})$, with the surface value $\mathrm{T}_{0}=258 / \mathrm{I}_{0}$. Lin also defined a crosslink depth $\left(\mathrm{z}^{*}\right)$ when the CXL efficacy reaching it maximum, given by $\mathrm{z}^{*}=\ln$ $\left(\mathrm{NE}_{0}\right) / \mathrm{A}$, with $\mathrm{N}=0.16$ (for $\mathrm{D}>>1 \mathrm{~cm}$ ) and $\mathrm{N}=0.224$ (for $\mathrm{D}=500 \mathrm{um}$ ). We note that $\mathrm{z}^{*}$ is an increasing function of the UV dose, but decreasing function of $\mathrm{A}_{\text {and }} \mathrm{C}_{0}$.

The UV light intensity increases from its initial value $I(z, t)=I_{0}$ $\exp [-\mathrm{A} 1 \mathrm{z}]$ to steady-state vale given by $\mathrm{I}(\mathrm{z}, \mathrm{t})=\mathrm{I}_{0} \exp [-\mathrm{A} 2 \mathrm{z}]$, with $A 1=2.3 a C_{0}+Q, A 2=2.3 b C_{0}+Q$. For $\mathrm{C}_{0}=0.1 \%, \mathrm{a}=204(1 / \mathrm{cm} / \%)$, $\mathrm{b}=50(1 / \mathrm{cm} / \%)$, and $\mathrm{Q}=32(/ \mathrm{cm})$, we obtain $\mathrm{A}_{1}=79(1 / \mathrm{cm})$, and $A_{2}=43.5(1 / \mathrm{cm})$, with an averaged value of $61(1 / \mathrm{cm})$, which are much larger than the RF-constant model with a value of 42.5 (1/ $\mathrm{cm})$. If one assumes $Q=b=0$, then $A=46.9(1 / \mathrm{cm})$, which is smaller than our averaged value of $61(1 / \mathrm{cm})$. Numerical simulation of Lin and Cheng [6], also showed another fit $A=2.3\left[\mathrm{mbC}_{0}+\mathrm{Q}\right]$, with $\mathrm{m}=1.5$ for $b=50(1 / \% / c m)$, which is fit to the CXL efficacy (at steady state). In this fitting, (for $D=500 \mathrm{um}$ ), $A=49$ and $66\left(1 / \mathrm{cm}\right.$ ) for $\mathrm{C}_{0}=0.1 \%$ and $0.2 \%$.

\section{Validation of Bunsen roscoe law (BRL)}

To shorten the CXL treatment duration while maintaining the similar CXL efficacy, accelerated CXL (AXL) protocols have been proposed to replace the Dresden low-power protocol based on the BRL of reciprocity [19] stating that the effect of a photo-biological reaction is proportional only to the total irradiation dose (E=It), or the product of intensity (I) and exposure time (t). To achieve the same efficacy, the required exposure time based on BRL is given by $t=E / I$, which gives the protocol for AXL; for example, $t=(30,10,5$, $3,2)$ minutes for $\mathrm{I}=(3,9,18,30,45) \mathrm{mW} / \mathrm{cm}^{2}$. Validation of BRL has been challenged by Lin's non-linear law and the S-formulas for CXL efficacy $[6,7,10]$. Wernli et al. [17] also pointed out the limitation of BRL due to the sudden drop of efficacy at UV intensity around $45 \mathrm{~mW} / \mathrm{cm}^{2}$. To improve the CXL efficacy, extended exposure time and/or dose, has been proposed $[20,21]$ to compensate the drawback of exposure time predicted by BRL. Moreover, a concentration-controlled method (CCM) was proposed by Lin [11] to improve the CXL efficacy by resupply of RF during the UV exposure.

\section{The role of oxygen and pulsed mode}

CXL efficacy is governed by both oxygen-mediated (OM) and non-oxygen-mediated (NOM) 3-pathway processes, rather than the conventionally believed type-II only (oxygen-mediated) mechanism $[9,10]$. Both type-I and type-II reactions can occur simultaneously, and the ratio between these processes depends on the type of photosensitizers (PS) used, the concentrations of PS, substrate and oxygen, the kinetic rates involved in the process, and the light intensity, dose, PS depletion rate etc. The CXL 3-pathway kinetics maybe described as follows. For type-I, the riboflavin triplet state [T] may interact directly with the stroma collagen substrate $[\mathrm{A}]$ under NOM (with a rate constant $\mathrm{k}_{8^{\prime}}$ pathway-1); or with the ground-state oxygen $\left[{ }^{3} \mathrm{O}_{2}\right]$ to form reactive oxygen species [0-] under $\mathrm{OM}$; and in type-II process, $[\mathrm{T}]$ interacts with $\left[{ }^{3} \mathrm{O}_{2}\right]$ to form a singlet oxygen $\left[{ }^{1} \mathrm{O}_{2}\right]$. [T] may also relax to riboflavin ground state (with a rate constant $\mathrm{k}_{5}$ ). Both reactive oxygen species (ROS), [0 $\left.\mathrm{O}^{-}\right]$and $\left[{ }^{1} \mathrm{O}_{2}\right]$, can either relax to $\left[{ }^{3} \mathrm{O}_{2}\right]$, or interact with $[\mathrm{A}]$ for crosslinking.

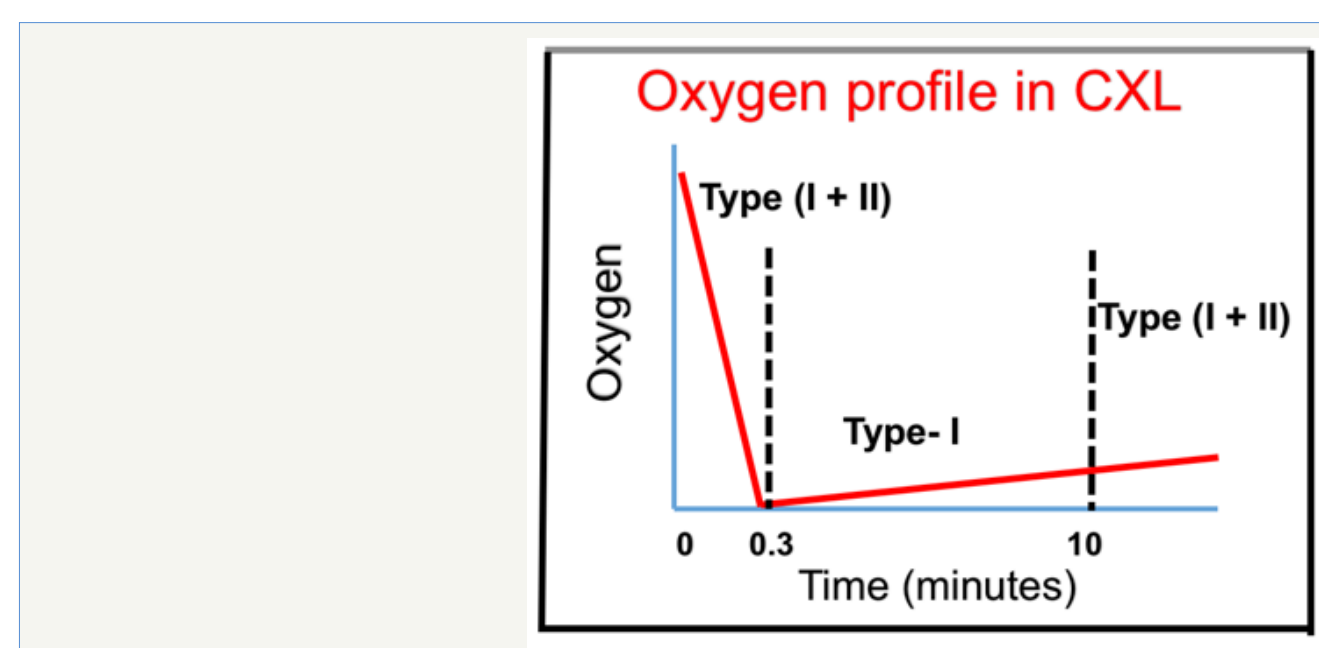

Figure 1: Schematics of the oxygen concentration profiles in CXL; in the transient stage, both type-I and type-II coexist until the oxygen is depleted; then type-I dominates before the oxygen is replenished $[9,10]$. 
Schumacher et al. [15] reported the NOM-type-I CXL, in contrast to Kling et al. [22] claiming that oxygen-mediated type-II played the critical role of CXL efficacy. Furthermore, Kamaev et al. [18] claimed that CXL is NOM-type-I dominant, while the OM-type-II only plays a limited and transient role, as shown by Fig. 2. If Kling et al. [5] were correct, then all the reported results of epi-on CXL would not be possible, since only limited and transient oxygen supply is available. Lin $[9,11]$ proposed mathematical model in supporting the claims of Kamaev et al. [18]. Pulsed mode was claimed to have higher efficacy than CW mode [22]. This conclusion, I believe, is due to clinical measured errors and/or non-controlled comparison of RF concentration during the UV exposure, based in Lin \& Kamaev studies $[9,18]$ that OM-type-II only plays a limited and transient role. As shown by (Figure 1), the role of oxygen resupply (and pulsed mode) takes few minutes. Therefore, pulsing in few seconds would not help the Type-II efficacy.

\section{Results and Discussion}

\section{Dresden vs. modern protocols}

The standard Dresden (SD) protocol was proposed by Wollensak et al. [2] in 2003, where a UVA light (at $365 \mathrm{~nm}$ ) was used to treat cornea $9 \mathrm{~mm}$ zone at an intensity of $3.0 \mathrm{~mW} / \mathrm{cm}^{2}$ for 30 minutes, delivering a fluence (dose) of $5.4 \mathrm{~J} / \mathrm{cm}^{2}$. Modern protocols, named as CCM by Lin [11], used a limited resupply of RF to eliminate the extra blocking effect due to over resupplied RF in Dresden protocol. CXL efficacy is influenced by multiple factors including, the UV light intensity, exposure period and dose, the initial concentration profiles of RF and oxygen, the quantum yield of the RF triplet state, the kinetic rate constants of RF (in type-I) and oxygen (in type-II).

Besides, the protocol procedures defining how the RF drops are applied pre-operatively and during the UV exposure are also important, because they define the initial, and intra-procedure
RF concentration profiles (or diffusion depth). For example, the frequency of RF drops (Fdrop) applied on the cornea after the UV is turned on, and the waiting period (Twait) for each RF drops instillation during the UV exposure. In the conventional Dresden protocol, Fdrop is about 5 to 10 times and Twait $=0$. In contrast, our proposed concentration-controlled method (CCM) uses Fdrop $=2$ to 4 (for RF replenishment) and Twait is 1 or 2 minutes (for enough diffusion depth, with $\mathrm{D}>150 \mathrm{um}$ ). Kling et al. [23] recently reported the use of $1.5 \mathrm{~mW} / \mathrm{cm}^{2}$ intensity for 30 minutes exposure (or $2.7 \mathrm{~J} /$ $\mathrm{cm}^{2}$ dose) has similar efficacy as that of $3 \mathrm{~mW} / \mathrm{cm}^{2}$ and 30 minutes exposure $\left(5.4 \mathrm{~J} / \mathrm{cm}^{2}\right.$ dose). This feature may be easily realized by our S-function which has an optimal dose predicted to be about 3 to $4 \mathrm{~J} / \mathrm{cm}^{2}$, and the $5.4 \mathrm{~J} / \mathrm{cm}^{2}$ (for $3 \mathrm{~mW} / \mathrm{cm}^{2}$ ) is certainly higher than the optimal value proposed by Lin's CCM $[9,11]$.

\section{Cut-off maximum intensity}

Controversial results for the corneal cross-linking (CXL) efficacy in standard low-power Dresden-CXL (DXL) and accelerated-CXL (AXL) have been reported [24-27]. In addition, various maximum (cutoff) UV light intensities were clinically reported by Hammer et al. [25] (18mW/ $\left.\mathrm{cm}^{2}\right)$, Webb et al. [26] $\left(34 \mathrm{~mW} / \mathrm{cm}^{2}\right)$, Bao et al. [27] $\left(30 \mathrm{~mW} / \mathrm{cm}^{2}\right)$, and Wernli et al. [17] $\left(45 \mathrm{~mW} / \mathrm{cm}^{2}\right)$. Webb et al. [26] believed that AXL has a shallower crosslink depth which results a lower efficacy in AXL than DXL. However, in most reported protocols, even similar pre-operative RF drops administration were performed, significant different cutoff light intensity and CXL efficacy were still observed [24-27]. Therefore, further analysis based on influencing factors during the UV exposure, other than the pre-operative Rf condition, are required. Bao et al. [27] and O'Brart et al. [28] reported that higher efficacy can be achieved by using a higher RF concentration, in consistent with the predicted role of Rf concentration by Lin [12].

\section{CXL efficacy}

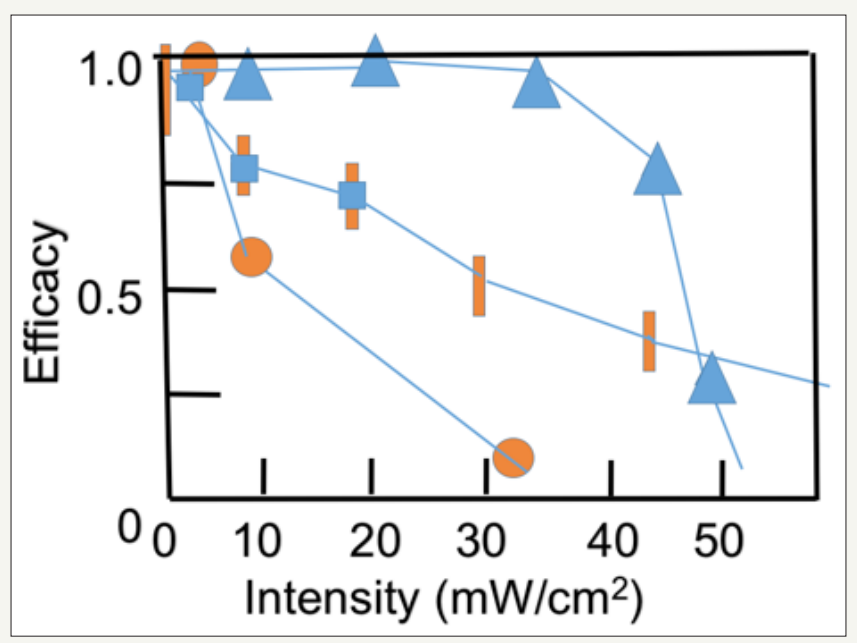

Figure 2: Efficacy vs. light intensity from clinical data of Wernli et al. [17] (triangles), Hammer et al. [25] (cubic), Bao et al. [27] (bar), and Webb et al. [26] (circle).

The type-I CXL efficacy is given by [6] Eff=1-exp(-S), where the S-function is proportional to $\left(\mathrm{FC}_{0} / \mathrm{I} 0\right) 0.5 \exp (0.5 \mathrm{Az})$, or (for $\mathrm{z}=0$ ) and stronger dependence of (for $\mathrm{z}>0$ ), because $\mathrm{A}$ is also proportional to $\mathrm{C}_{0}$. given by $A=290 m(1-0.25 z / D) C_{0}+32$ (with 
fit parameter m). Figure 2 shows the efficacy vs. light intensity from clinical data of Wernli et al. [17] (triangles), Hammer et al. [25] (cubic), Bao et al. [27] (bar), and Webb et al. [26] (circle). (Figure 3) shows the CXL efficacy versus riboflavin concentration shows the nonlinear feature, where theoretical curve (red curve) is compared with the clinical data (bars) of O’Brart et al. [28] (Figure 2 \& 3).

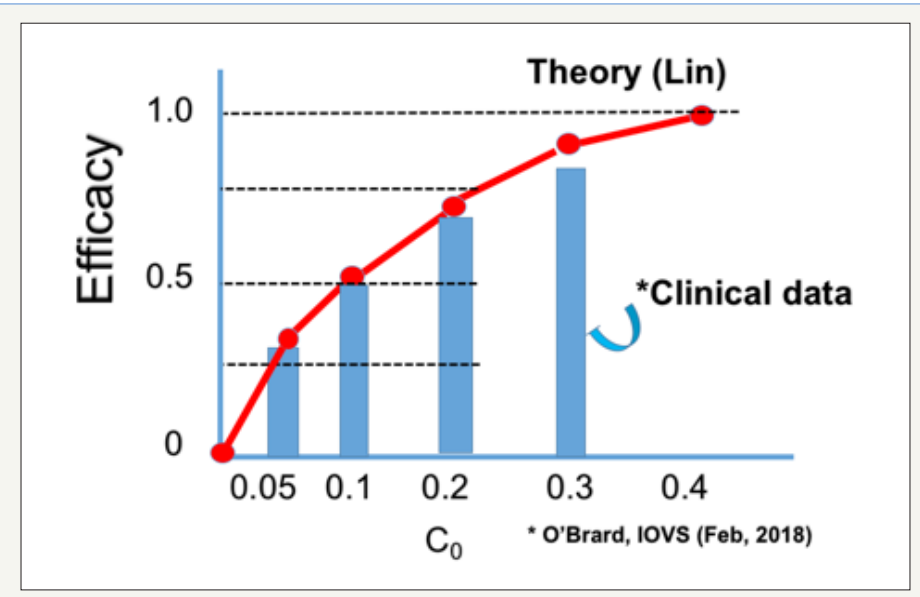

Figure 3: CXL efficacy versus riboflavin concentration shows the nonlinear feature, where theoretical curve (red curve) is compared with the clinical data (bars) of O’Brart et al. [28].

\section{Update on lin's CCM}

The intrinsic efficacy drawbacks of AXL, under a non-controlled situation in Dresden protocols, may be overcome by a newly proposed protocol called controlled-concentration-method (CCM) by Lin [11], in which extra Rf drops are applied to the cornea, at every crosslink time ( $\left.\mathrm{T}^{*}\right)$, during the UV exposure, having a frequency defined by Fdrop $=\mathrm{N}-1$, with $\mathrm{N}=0.365\left[\mathrm{I}_{0} / \mathrm{C}_{0}\right]^{0.5}$, where $\mathrm{N}=2$, for the referenced point with $\mathrm{I}_{0}=3 \mathrm{~mW} / \mathrm{cm}^{2}$ and $\mathrm{C}_{0}=0.1 \%$. Therefore, Fdrop $=(1,2,4,5,7)$, for $I_{0}=(3,9,18,30,45) \mathrm{mW} /$ $\mathrm{cm}^{2}$ and for $\mathrm{C}_{0}=0.1 \%$; which reduced to Fdrop $=(1,1,2,3,4)$, for higher $\mathrm{C}_{0}=0.3 \%$. Given Fdrop=N-1, the combined efficacy defined as $c-C e f f=1-\exp [-(S 1+S 2+\ldots S j)]$, with $\mathrm{j}=\mathrm{N}=\mathrm{Fdrop}+1$. The above Fdrop plays the critical factor influencing the CXL efficacy. Therefore, comparison of DXL and AXL without specifying the exact Fdrop-protocol is questionable. WE note that in the CCM, the time to resupply Rf drops is given by $\mathrm{T}^{*}$ which is a deceasing function of the light intensity. In comparison, the Dresden protocol (DXL), Rf drops were continuously administrated during the UV exposure, every 3 to 5 minutes, a fixed time regardless the light intensity.

For a low intensity $\left(3 \mathrm{~mW} / \mathrm{cm}^{2}\right)$ in DXL, the total exposure time is 30 minutes, therefore it has Fdrop $=6$ to 10 , which is overdone and will reduce the effective dose approximately $25 \%$ to $30 \%$ out of the $5.4 \mathrm{~J} / \mathrm{cm}^{2}$. In the protocol of Bao et al. [27] and Webb et al. [26], they did not resupply any Rf drops in their high intensity studies, corresponding to Fdrop $=0$ (or $N=1$ ). Therefore, their concluded comparison between DXL and AXL did not have a fair reference, and their AXL efficacy is lower than what are predicted by CCM, which has the maximum efficacy for AXL. The results of Spadea et al. [20,21], based on resupply of Rf drops (during the UV exposure) every 3 and 5 minutes, which showed similar efficacy of 3 and $9 \mathrm{~mW} / \mathrm{cm}^{2}$. Lin's S-formulas also demonstrates that epi-on CXL (having a smaller D and $\mathrm{C}_{0}$ ) is less efficient than epi-off CXL, in consistent with reported data of Spadea et al. [20].

\section{Crosslink volume}

A new CXL efficacy was proposed by Lin [12] given by the product of [strength] (or the maximal value of S) and the [depth] (or $\mathrm{z}^{*}$ ), i.e., the volume of stroma being cross-linked. It should be noted that deeper CXL (or larger $\mathrm{z}^{*}$ ) may be achieved by larger fluence $\left(E_{0}\right)$, i.e, more superficial CXL in higher $\mathrm{C}_{0}$ may be compensated by larger light-dose. However, considering optimal CXL with minimal UV exposure time (or dose), one requires an optimal range of $\mathrm{C}_{0}$ $0.15 \%$ to $0.3 \%$ and and $E_{0}=3.5$ to $4.5 \mathrm{~J} / \mathrm{cm}^{2}$, such that [depth] $\mathrm{z}^{*}=200$ to $300 \mathrm{um}$, with [strength] $\mathrm{S} 1=1.5$ to 2.0 (or CXL efficacy 1-exp(-S1) $=0.78$ to 0.86$)$, noting that high $C_{0}$ causes a competing of [strength] and [depth] which needs to be optimized. Greater detail with numerical simulation will be presented elsewhere.

\section{Demarcation line depth and CXL efficacy}

Spadea et al $[20,21]$ studied the relationship between CXL efficacy and the UV light exposure duration, intensity, and the demarcation line (DL) depth based on available measured data. They concluded that the duration of the procedure might be the rate-limiting step in the efficacy of CXL. The sudden-drop of DL depth at high intensity $\left(>45 \mathrm{~mW} / \mathrm{cm}^{2}\right)$ has the similar feature as the AXL efficacy reported by Wernli et al. [17]. However, it is clinically unclear whether DL depth is proportional to the crosslink depth or the CXL efficacy. Moreover, controversial results of AXL efficacy were reported due to inconsistent protocols which were not optimized.

The measured DL depth may be compared with a combined CXL efficacy given by c-Ceff=1-exp (-4R), with $R=\left(62 / t^{\prime}\right)\left[C_{0} F(z) / I_{0}{ }^{3}\right]^{0.5}$ , where $F(z)=1-0.5 z / D$, is the Rf initial concentration profile defined by a diffusion depth (D); $t^{\prime}$ is the resupply Rf drops every $t^{\prime}$-minutes. $\mathrm{R}$ is the efficacy ratio between the non-controlled Dresden protocol and the optimal protocol (via CCM) having $\mathrm{R}=1.0$, and $\mathrm{c}$-Ceff $=0.98$, for all range of UV intensity $\mathrm{I}_{0}=3$ to $60 \mathrm{~mW} / \mathrm{cm}^{2}$. Figure 2 shows the theoretical Curves $(1,2,3,4)$ calculated from the combined efficacy 
(c-Ceff) formula, with time of resupply $\mathrm{Rf}$ drops $\mathrm{t}^{\prime}=(4,2,1,0.5)$ minutes; noting that smaller $\mathrm{t}^{\prime}$ associated with larger Fdrop, therefore, has higher efficacy.

(Figure 4) may be compared with (Figure 2) (measured efficacy data). Comparing the theoretical curves and the measured DL depth, one may conclude that CXL efficacy is proportionally related to the DL depth, and both have a cutoff maximum intensity, under the non-controlled Dresden protocol, as reported by Wernli et al. [4]. It should be noted that the theoretical Curve- 4 (with $t^{\prime}=0.5$ minutes, or Fdrop=3) gives the optimal efficacy similar to CCM [11] (Figure 4).

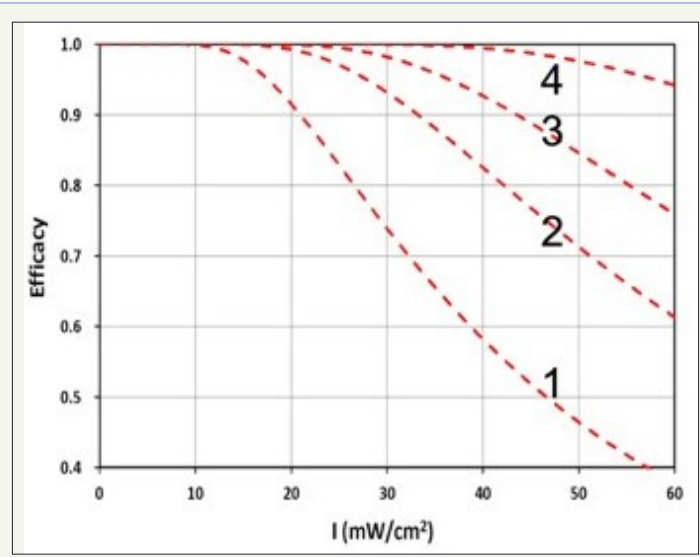

Figure 4: Theoretical CXL efficacy vs. UV light intensity for resupply of RF drops at various time, $t^{\prime}=(4,2,1,0.5)$ minutes, for Curves $(1,2,3,4)$

\section{Conclusion}

We have presented the resolutions of controversial issues in CXL via factors influencing the CXL efficacy. To improve the efficacy of ACXL, a CCM was proposed. A new CXL efficacy defined by the product of [strength] (or the maximal value of S) and the [depth] (or $z^{*}$ ), i.e., the volume of stroma being cross-linked is introduced. CXL efficacy is proportionally related to the DL depth, and both have a cutoff maximum intensity.

\section{References}

1. Wollensak G, Spoerl E, Wilsch M, Seiler T (2003) Endothelial cell damage after riboflavin-ultraviolet-A treatment in the rabbit. J Cataract Refract Surg 29(9): 1786-1790.

2. Hafezi F, Randleman JB (2016) Corneal collagen cross-linking. Slack Inc, NJ, USA.

3. Lin JT (2015) Analytic formulas on factors determining the safety and efficacy in UV- light sensitized corneal cross-linking. Invest Ophthalmol Vis Sci 56: 5740-5741.

4. Lin JT (2015) On the dynamic safety for cross linking in thin corneas (350-398 um) with extra protection under a contact lens. J Refract Sur 31(7): 495-496.

5. Lin JT, Cheng DC, Chang C, Yong Zhang (2015) The new protocol and dynamic safety of UV-light activated corneal collagen cross-linking. Chinese J Optom Ophthalmol Vis Sci 17: 140-147.

6. Lin JT, Cheng DC (2017) Modeling the efficacy profiles of UV-light activated corneal collagen crosslinking. PloS One 12: e0175002.

7. Lin JT (2017) Efficacy and $Z^{*}$ formula for minimum corneal thickness in UV-light crosslinking. Cornea 36(11): 30-31.

8. Lin JT (2017) Photochemical Kinetic modeling for oxygen-enhanced UVlight-activated corneal collagen crosslinking. Ophthalmology Research $7(1): 1-8$

9. Lin JT (2018) Efficacy S-formula and kinetics of oxygen-mediated (type-II) and non-oxygen-mediated (type-I) corneal cross-linking. Ophthalmology Research 8(1): 1-11
10. Lin JT (2018) A Critical review on the kinetics, efficacy, safety, nonlinear law and optimal protocols of corneal cross-linking. J Ophthalmology \& Visual Neuroscinece 3: 017.

11. Lin JT (2018) A proposed concentration-controlled new protocol for optimal corneal crosslinking efficacy in the anterior stroma. Invest Ophthalmol Vis Sci 59(1): 431-432.

12. Lin JT (2018) The role of riboflavin concentration and oxygen in the efficacy and depth of corneal crosslinking. Invest Ophthalmol Vis Sci 59 (in press).

13. Lin JT (2018) Corneal Collagen Cross-linking (CXL): Controversy and Fundamentals. Ophthalmol Clin Res 1(1): 17-21.

14. Mooren P, Gobin L, Bostan N, Wouters K, Zakaria N, et al. (2016) Evaluation of UVA cytotoxicity for human endothelium in an ex vivo corneal cross-linking experimental setting. J Refract Surg 32((1): 41-46.

15. Schumacher S, Mrochen M, Wernli J, Bueeler M, Seiler T (2012) Optimization model for UV-riboflavin corneal cross-linking. Invest Opthamol Vis Sci 53(2): 762-769.

16. Kling S, Hafezi F (2017) An algorithm to predict the biomechanical stiffening effect in corneal cross-linking. J Refract Surg 33(2): 128-136.

17. Wernli J, Schumacher S, Spoerl E, Mrochen M (2013) The efficacy of corneal cross-linking shows a sudden decrease with very high intensity UV light and short treatment time. Invest Ophthalmol Vis Sci 54: 11761180 .

18. Kamaev P, Friedman MD, Sherr E, Muller D (2012) Photochemical kinetics of corneal cross-linking with riboflavin. Invest Ophthalmol Vis Sci 53: 2360-2367.

19. Bunsen RW, Roscoe HE (1862) Photochemical researches-Part V. On the measurement of the chemical action of direct and diffuse sunlight. Proc R Soc Lond 12: 306-312.

20. Spadea L, Genova LD, Tonti E (2018) Corneal stromal demarcation line after 4 protocols of corneal crosslinking in keratoconus determined with anterior segment optical coherence tomography. J Cataract Refract Surg 44(5): 596-602.

21. Spadea L, Tonti E, Vingolo EM (2016) Corneal stromal demarcation line after collagen cross-linking in corneal ectatic diseases: a review of the literature. Clin Ophthalmol 10: 1803-1810. 
22. Kling S, Hafezi F (2017) An algorithm to predict the biomechanical stiffening effect in corneal cross-linking. J Refract Surg 32(2): 128-136.

23. Kling S, Hafezi F (2017) Biomechanical stiffening: Slow low-irradiance corneal crosslinking versus the standard Dresden protocol. J Cataract Refract Surg 43(7): 975-979.

24. Toker E, Cerman E, Oscan DO, Seferoglu OB (2017) Efficacy of different accelerated corneal crosslinking protocols for progressive keratoconus. J Cataract Refract Surg 43(8): 1089-1099.

25. Hammer A, Richoz O, Mosquera SA, Tabibian D, Hoogewoud F, et al (2014) Corneal biomechanical properties at different corneal crosslinking (CXL) irradiances. Invest Ophthalmol Vis Sci 55(5): 2881-2884.
26. Webb JN, Su JP, Scarcelli G (2017) Mechanical outcome of accelerated corneal crosslinking evaluated by Brillouin microscopy. J Cataract Refract Surg 43(11): 1458-1463.

27. Bao FJ, Zheng YR, Liu C, Zheng X, Zhao Y, et al. (2018) Changes in corneal biomechanical properties with different corneal cross-linking irradiances. J Refract Surg 34(1): 51-58.

28. O'Brart NAL, O'Brart DPS, Aldahlawi, Hayes S, Meek KM (2018) An investigation of the effects of riboflavin concentration on the efficacy of corneal cross-Linking using an enzymatic resistance model in porcine corneas. Invest Ophthalmol Vis Sci 59(2): 1058-1065.
For possible submissions Click Here

Submit Article

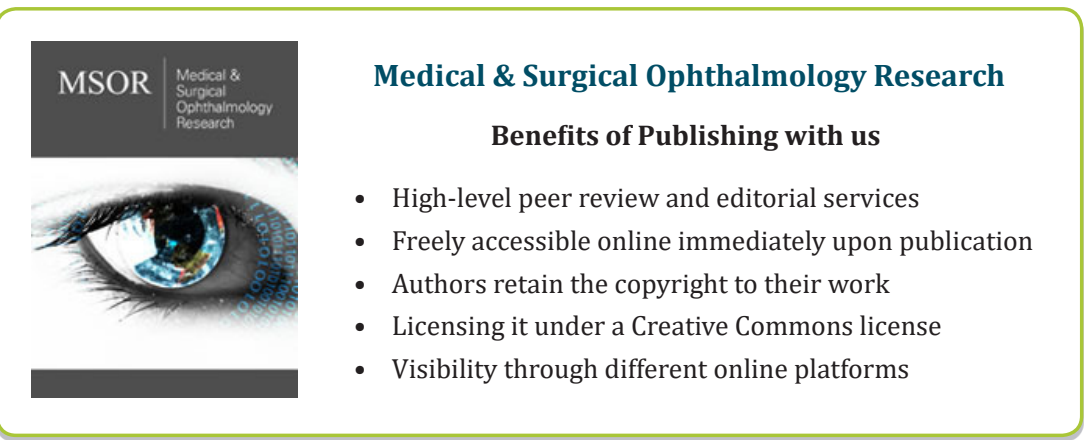

